1 The application of Psychologically Informed Practice: Observations of experienced

2 physiotherapists working with people with chronic pain.

4 Diarmuid Denneny $^{1}$, Annina Frijdal (nee Klapper) ${ }^{2}$, Nadia Bianchi-Berthouze ${ }^{2}$, Jim

5 Greenwood $^{1}$, Rebecca McLoughlin ${ }^{1}$, Katrine Petersen ${ }^{1}$, Aneesha Singh ${ }^{2}$, Amanda C. de C

$6 \quad$ Williams ${ }^{2}$

7 1. UCLH NHS Foundation Trust, London, UK

$8 \quad$ 2. University College London, UK

11 Abstract.

12 Objectives: Psychologically informed practice (PIP) is advocated for physiotherapists to help

13 people with chronic pain. There is little research observing how PIP is delivered in clinical

14 practice. This study describes behaviours and techniques used by experienced physiotherapists

15 working with groups of people with chronic pain.

16

17 Setting and Participants: Experienced physiotherapists $(n=4)$ were observed working with

18 groups of people with chronic pain in out-patient pain management, and physiotherapy

19 departments, in a large UK city centre teaching hospital.

21 Design: We observed the clinical behaviours and interpersonal skills of experienced

22 psychologically informed physiotherapists, enriched by their accounts of intentions. The

23 physiotherapists were audio and video recorded delivering group movement sessions. 
24 Recordings were reviewed with the physiotherapists for elaboration of intentions, then

25 thematically analysed for comparison with defined CBT competencies.

27 Results: Four themes representing physiotherapist intentions when working with people with

28 chronic pain were identified; building a therapeutic alliance, reducing perceived threat,

29 reconceptualising beliefs and somatic experience, and fostering self-efficacy. The

30 physiotherapists also reflected on challenges including engaging patients in self-management,

31 encouraging activity and reinforcing rather than correcting movement. Considerable overlap

32 existed between the observed behaviours in this study and existing CBT competencies.

33 Conclusions: This paper complements current recommendations for delivering psychologically

34 informed physiotherapy by providing examples of these skills being used in clinical practice.

35 Further research supporting the development of training for, and mentoring of,

36 physiotherapists, to promote competence and confidence in delivering psychologically informed

37 interventions is recommended.

39 Key words:

40 Chronic pain, Psychologically informed, Cognitive Behavioural Therapy, Qualitative

\section{Contribution of paper}

43 - This study describes behaviours and techniques used by experienced physiotherapists

$44 \quad$ working with groups of patients with chronic pain.

45 - This paper complements current recommendations for delivering psychologically 
informed physiotherapy by providing examples of these skills being used in clinical practice.

\section{Background:}

54 Chronic pain is difficult to treat and poses a major healthcare challenge, affecting up to half the

55 UK population [1]. Its management requires a biopsychosocial model prioritising self-

56 management [2], since treatment of even the most severely affected $1 \%$ requires more

57 resources than could ever be available [3]. Psychological approaches to extend and enhance the

58 skills of physiotherapists, and promote self management with patients, have been advocated for

59 over twenty years [4]. Delivering these psychological approaches and promoting patient self-

60 management necessitates changes in usual behaviours of health care practitioners [5].

62 Guidelines recommend treatment packages with psychologically informed practice alongside

63 exercise and activity [6,7], but do not specify the interpersonal skills and behaviours required by

64 clinicians to deliver this approach. The phrase psychologically informed practice (PIP) [2]

65 represents a trend towards inter-professional working [8,9], particularly in the management of

66 chronic pain (see McLoughlin [10] for example). The term PIP is itself open to interpretation.

67 Main and George [2] focus on the patient context and experience, referring "primarily to the 
68 inclusion of a specific focus on psychosocial or psychological factors (both clinical and

69 occupational) for [chronicity] risk determination and as potential targets for intervention by the

70 physical therapist". Wilson [11], by contrast, describes PIP in terms of the methods used by the

71 physiotherapist, delivered "within a psychological framework". PIP is often taken to involve

72 cognitive-behavioural techniques (CBT), which may encompass mindfulness and/or acceptance-

73 based interventions, stress management, relaxation training, hypnosis, coping skills training,

74 problem solving, systematic desensitisation, and motivational interviewing, in combination with

75 physiotherapy and delivered by a physiotherapist $[, 12,13]$.

1.

79 Physiotherapists have the training and credibility to assess risk versus safety in human

80 movement and have a key role in reducing disability in persistent pain conditions [14]. In

81 healthcare delivery they provide a bridge between biomedical and psychosocial models of care,

82 and many interactions with patients provide education, advice and reassurance intending to

83 target unhelpful beliefs and behaviours [14].

84

85 While recognising that other models of behaviour change are available [15], here we focus on

$86 \mathrm{CBT}$, as a pervasive model of effective change in chronic pain rehabilitation. CBT draws on a

87 theoretical and evidence base: it is practiced flexibly, in the moment, using understanding of the

88 principles and applying them to physiotherapeutic content. Identification of unhelpful beliefs or

89 thinking patterns, and of ways to work with them in a physiotherapeutic setting, have been 
90 described in the physiotherapy literature $[16,17]$. CBT requires a collaborative relationship with

91 the client, sharing framework and methods, as a joint exploration in which the client learns skills

92 to apply beyond the therapeutic setting in development of self-management skills. Experiential

93 learning is recognised as fundamental to the behaviour change required for self-management

94 skills [15]. Physiotherapists are ideally placed to promote experiential learning particularly

95 through movement and activity focused sessions.

96 Relevant competences in CBT for psychologist practitioners are shown in table 1, and share

97 characteristics with those outlined by Hansen [18] and the Physiotherapy Pain Assocation [19]

98 for physiotherapists working in pain services.

99

\section{Table 1: Competence model for cognitive and behavioural therapy (CBT) practitioners}

101 Through undergraduate and early career training, physiotherapists are expected to demonstrate

102 basic physiotherapy competencies that align with generic and basic CBT competences identified

103 in Table 1 (I. and II.), such as the ability to engage the client, foster a good relationship, agree

104 goals, and set homework. Newly qualified physiotherapists are expected to be able to prescribe

105 appropriate and engaging home exercise programmes for patients.

106

107 Many factors influence the patient/therapist relationship and treatment including; therapeutic

108 alliance [20], practitioner/therapist effects [21], attitudes and beliefs [22], empathy [23,24], and

109 other emotional responses [25]. While many physiotherapists demonstrate a positive attitude

110 and beliefs regarding PIP, they identify the need for further training to instil confidence in 
111 practice [26]. Physiotherapists have reported low confidence in challenging unhelpful thoughts

112 [18]. Interpersonal skills used to deliver PIP are poorly specified in the literature $[27,28]$ and

113 physiotherapists tend to prefer dealing with the more mechanical aspects of low back pain [22],

114 and consider that neither their initial training nor available professional development training

115 provided them with the requisite skills and confidence to successfully identify and address the

116 psychological and social aspects of low back pain [22]. Where skills training has subsequently

117 been sampled in practice, it has not shown as consistent or widespread effects as intended [29].

119 Thus while there is widespread enthusiasm for the aims of PIP, within the profession and the

120 NHS, we are still some way from defining the competences of PIP, and how best to train them.

121 Rather than training physiotherapists in researcher-selected elements of CBT, and assuming that

122 the physiotherapists practiced it effectively with patients, this paper rather examined what

123 psychologically informed physiotherapists actually do in practice, taking a phenomenological

124 approach to their behaviours and the intentions informing these behaviours, recorded during

125 sessions with patients with chronic pain.

128 Methods

130 Clinical behaviours and interpersonal skills were collected from observations, enriched by

131 participants' accounts of their intentions, and mapped against the CBT competences identified 
132 in table 1. NHS ethics approval was obtained (Ref 11/078).

\section{Participants and recruitment}

136 Participating physiotherapists (table 2) were recruited from a large central London teaching

137 hospital as part of research on the design of technology to support self-directed rehabilitation

$138[30,31,32]$. They were known to the research team, having advised on other aspects of the

139 larger research study. The observed therapists had variable training but considerable experience

140 working alongside psychologists in chronic pain. All physiotherapists who were approached

141 agreed to take part and no drop outs occurred. Partaking in the study was of their own valition.

142 In order to be included in the study physiotherapists had to:

143 - run a group treatment for chronic pain patients over several weeks

144 - have at least 5 years of expertise within the field of chronic pain

145 - be a member of the medical team of the UCLH or NHNN

147 Table 2: Participating physiotherapists

148 The observed group sessions varied, but were all exercise based and did not include any manual 149 therapy (Table 3).

150

151 Table 3: Summary of the groups led by physiotherapists observed. 


\section{Data collection}

154 Physiotherapists were video and audio-recorded, with their consent and that of patients.

155 Cameras, and a small wearable microphone, were used to record facial expressions and body

156 movement and to capture physiotherapists' communicative and expressive behaviours [33].

157 Recordings were then reviewed with the physiotherapists for elaboration of intentions, then

158 thematically analysed for comparison with defined CBT competencies [34].

159 Initially, physiotherapists reviewed the video with a psychologist researcher (AF), using

160 'naturalistic social cognition methodology' [34]. The researcher (female) was a psychology

161 graduate, new to research in pain management, with a 6 month postgraduate scholarship

162 interest in influences of social interaction on pain, There was no script for these one-off

163 interviews which lasted for approximately one hour and no pilot interviews were undertaken.

164 The video was stopped by either party to explore or explain behaviour and intentions in

165 interactions with patients, in particular, use of CBT techniques. The process was audio-recorded

166 and transcribed verbatim, which enabled a deep exploration of the physiotherapists'

167 understanding of situations and decision-making processes at particular moments during

168 treatment.

\section{Data analysis}

170 Thematic analysis was applied to the transcripts, an inductive process driven by data content

171 and involving an iterative and reflexive process of extracting superordinate themes from the raw

172 data, which was managed using Microsoft Excel (2007), according to established methods

$173[35,36]$. Although both researchers who conducted the thematic analysis were psychologists (AF

174 and $\mathrm{AW}), \mathrm{CBT}$ competences were not accessed until thematic analysis was complete. The 
175 researcher $(\mathrm{AF})$ arranged individual meetings with each participating physiotherapist where

176 summaries of the analysis, and the opportunity to feedback, were given.

177

178 Results

179

180 Initial coding generated 112 behaviours and intentions; these were grouped thematically and

181 collapsed to some extent where intentions of behaviours were similar, a process carried out by

182 the Interviewer (AF) and subsequently by another author (AW). From this, four themes

183 representing physiotherapist intentions emerged, incorporating 11 subthemes, each described

184 by specific behaviours, some of which appear in more than one theme. Behaviours concerning

185 general process events such as catching a patient's eye to gain their attention or to indicate

186 interest or approval, were not categorised.

187

188 The four themes were:

189 1. building a therapeutic alliance

$190 \quad$ 2. reducing perceived threat

1913 3. reconceptualising beliefs and somatic experience

192 4. fostering self-efficacy

193 Examples of strategies used by the therapists under each theme/subtheme are given below as

194 well as one representative quotation. For further illustrative quotations see table 4.

195

196 Theme 1 Building a therapeutic alliance 
198 Theme 1 consisted of various behaviours directed towards building a therapeutic alliance,

199 including repeating patients' words to modify or emphasise important points; copying or

200 demonstrating movement; inclusive language; nonverbal behaviour; and humour. All behaviours

201 were directed towards engaging patients and establishing trust. We identified no subthemes in

202 this category.

B: "So often I try and repeat the actual words that people have said, to acknowledge hearing them, and to kind of mirror... you know, I have heard what you have said and reinforce that that's a helpful thing that they have said."

\section{Theme 2: Reducing perceived threat}

209 We identified behaviours including goal-setting, problem-solving, and use of activity monitoring

210 in this theme, with two sub-themes:

\section{2 i) Normalising somatic sensations:}

214 Strategies used by physiotherapists included explaining somatic experience; reinterpreting

215 symptoms as unthreatening; focusing on positive aspects and sensations; reminders of

216 breathing; and even speaking tone and relaxed manner to model calm.

D: "When they're moving and stretching and they hear clicking it can be quite frightening, so it's just to remind them these are normal sounds. We will have talked about what they are, what clicking is, what's going on in the body previously, so that I

222 ii) Matching demand to patient tolerance: 
224 The physiotherapist provided individual options or modifications for exercises, or suggested

225 taking a break, in order to mitigate patient anxiety and ensure participation.

D: "There is a bit of myth that exercise has to be done a certain way or it's not right, and actually there is a spectrum and there is lots of ways you can do, so it's finding what works for you. So it's giving.... giving options, and then the patient or the person can choose what works best for them."

\section{Theme 3: Reconceptualising beliefs and somatic experience}

Helping the client to identify and modify assumptions and rules was evident in this theme, as was planning and conducting tasks to identify any bariers to activity, often referred to as behavioural experiments. We identified three sub themes.

\section{i) Using behaviour to influence beliefs}

Here physiotherapists used what was happening in the moment to improve patient understanding; drew on patients' experiences for illustrating behaviours; set up behavioural experiments to demonstrate principles; provided options for achieving same end by different means; guided attention; recorded and reflected on activity.

B:"Sometimes ... I might get to the point where ... we're just getting stuck, so I might go more into... "How about we set up an experiment or how about you give an alternative approach and give this a go and just see what happens, and it might be that your way is a better way, but you might like the new way." 


\section{ii) Direct engagement with beliefs and changing beliefs}

251 Physiotherapists at times took a more direct approach: outlining facts; rephrasing a patient

252 question or comment for discussion; asking about concerns; providing information about

253 behavioural options and their consequences; asking the patient to describe behaviours and

254 their consequences in context and generating alternative possible behaviours with

255 consequences; negotiating goals and expectations; summarising learning from session;

256 repeating key concepts.

D: "Should we sit with crossed legs?

Patient 1: No.

Patient 2: Yes.

D: Should we not sit with crossed legs? Is crossed legs sitting bad for us?

Several patients: Yes.

D: They are used to me just saying "Is that a bad thing? Why is that a bad thing?" 'Cause it's getting them to question what they have been told by others. It's amazing how much we just take as gospel ... rather than really thinking about it and exploring it yourself and working out why it is that way."

In some cases, this took the form of eliciting patient reflection in order to change beliefs.

C: "There is a whole issue around, 'Oh this consultant doesn't want to see me again', 'Why is he telling me to go away?' kind of thing, and then asking them... see if they can come up with any answers that are a little bit more constructive than 'The medical service is letting me down'. Sometimes if you ask a patient, "So why do you think the consultant hasn't rebooked you?" or whatever, they might say, 'Well, I don't think he can help me really'. You know, if they can come up with those answers, 'Well, maybe it's because I need to live with it...' or something... towards that sort of acceptance. ... so instead of feeling unfairly treated, they might come to a little bit more of acceptance: 'Okay, the doctor does really care about me, but actually he hasn't got any medical treatment that would be beneficial for me', which is much more helpful."

\section{iii) Recognising achievement:}


282 Physiotherapists put emphasis on recognising achievements by recording activity and exercise;

283 prompting attention to aspects of movement that represent achievement; and reminders of

284 short term goals.

285

286

287

288

289

290

291

292

293 The emphasis on engaging and supporting the client in self-management was particularly

294 evident in this theme, with four sub themes.

C: "If we tell the patient "Next stretch you're going to have to bend forward", for example, you might already trigger off some areas in their brains that go, "Ooooh, bending forwards is really bad", but if you just start doing it and then talk through it as you're doing it ... there's something that says to them, "Oh, actually I am bending!"

\section{Theme 4: Fostering self-efficacy}

evident in this theme, with four sub themes.

295

297

298 Physiotherapists used problem-solving with patient; elaborating description to involve or

299 normalise; and focusing on experience not performance.

\section{i) Resolving possible barriers to activity,}

C: It doesn't sound silly at all, they are completely normal thoughts that you just want to walk down the street and not be noticed... These are absolute classic obstacles to people pacing: "I want to be normal. I don't want to stop and catch my breath". 
This consisted of encouraging any movement over 'correct' movement; demonstrating

308

309

310

311

312

313

314

315

exercises; avoiding making instructions too specific; resisting requests for prescriptive advice about exercise; modelling the patient making his/her own choices about activity; getting the patient to pace stretches by counting breaths.

D: [re giving choices of exercise] "We all want to have choice and want to feel like we are
a bit more in control of things." iii) Encouraging patient autonomy,

Patient autonomy was guided by physiotherapists avoiding eye contact to reduce patients'

dependence on supervision; moving away from patient; shifting topic or starting new

conversation; deciding when to explain more and when to stop talking; reminders of short term goals; asking patients to lead parts of sessions.

A: "I think sometimes if you're too prescriptive, people will think that... they can only exercise with supervision and then you're over-medicalising them again and a whole part of this process is to de-medicalise it."

\section{iv) Encouraging self-attribution of success}

Physiotherapists emphasised self-attribution of success by giving positive feedback to the whole group; recording activity; pausing to reflect on achievements; and giving feedback to individual about doing something challenging.

C: "The ultimate aim is for them to move with confidence rather then moving in a way that we want them to move." 
334 Several comments from each physiotherapist referred to behaviours used in combination, but

335 more striking were dilemmas that occurred across themes, in three particular areas:

All these were particularly demanding in group sessions, and often not possible for individual

physiotherapists to resolve although they clearly reflected critically on their decisions.

1. Whether they succeeded in engaging a patient in learning self-management methods, rather than eliciting compliance through instructions.

2. Rather than listen to the patient's concerns, and possibly attempt to resolve those that undermined movement, shouldthe physiotherapist encourage any activity, even to 'try it and see'..

3. When to reinforce any movement and effort rather than trying to correct or shape the particular movement towards the desired performance. 
them and share understanding, on a firm basis of their psychological stance on patients' difficulties.

\section{Discussion}

Recommendations for the use of psychologically informed approaches alongside physical

372 psychological therapies [37]. To date, however, very little is published on the actual clinical

373 behaviours of experienced physiotherapists working in a psychologically informed way with

374 people who have chronic pain. This paper provides clinical examples of how experienced

375 physiotherapists deliver this approach and how their behaviours link to an existing competency

376 framework.

\section{Mapping observed behaviours with CBT competencies}

380 Considerable overlap existed between the observed behaviours in this study and the CBT

381 competencies presented in table 1 . The capacity to work from the patient's perspective is

382 evident across all themes, engaging with patients' beliefs and current level of activity or

383 willingness to make a particular movement. Building a therapeutic alliance, which emerged as 
384 the first theme, was perhaps the longest-established skill of these physiotherapists, not

385 acquired only through CBT training and supervision. It was described in an integrated way

386 across verbal and nonverbal behaviours, with (perhaps surprisingly) no subthemes. Evidence of

387 CBT metacompetences, including use of clinical judgement in implementing treatment, adapting

388 interventions to the client, and using and responding to humour, emerged across all themes.

390 Throughout themes two and three (Reducing perceived threat and Reconceptualising beliefs

391 and somatic experience) there is evidence of brief, informal behavioural experiments being

392 integral to PIP sesssions. Together with review and reflection, demonstrated largely in themes

393 three and four (Reconceptualising beliefs and somatic experience and Fostering self-efficacy),

394 our evidence shows PIP sessions embody experiential learning. To our knowledge this is the

395 first account of experienced physiotherapists demonstrating the application of CBT and the

396 importance of behavioural experimentation. By building a therapeutic alliance and reducing

397 perceived threat, the physiotherapists were able to facilitate the reconceptualisation of beliefs

398 and experience and enhance self-efficacy.

400 Physiotherapists use a range of psychological skills with patients, but reflecting on their

401 performance in a way that helps to develop competence is often attenuated by time pressures,

402 reimbursement issues, lack of appropriate supevision, patients' expectations of physiotherapy,

403 and other barriers [16]. Reflection is an important tool for the therapist, in terms of using

404 strategies they have learned in training to enhance practice. Within this study, physiotherapists

405 reflected that at times "they were uncertain whether they had succeeded in engaging a patient 
407 physiotherapy". This quote illustrates that psychologically informed physiotherapists are aware

408 of the importance of reflecting on an action with the patient, identifying what has been learned 409 and how this will influence future behaviour. .

411 Three of the four physiotherapists in the study had attended at least a two day CBT training

412 course and had monthly psychology supervision. None of the CBT courses included follow-up

413 supervision or supported reflection, despite current recommendations for training

414 physiotherapists in PIP [13] and evidence demonstrating improving learning [38]. This highlights

415 an important gap in current PIP training and delivery, supporting integration of theoretical skills

416 in practice, especially where psychology supervision is not accessible. The development of

417 mentoring and peer supervision networks may provide some ways to address this issue.

\section{Strengths and limitations}

421 The real-life material used offers direct evidence of what psychologically-informed

422 physiotherapists do in practice, distinct from role-play or discursive accounts. However,

423 physiotherapists may have been influenced by the presence of cameras and the psychologist

424 operating them, and made more conscious effort to display CBT competencies in their

425 interactions with patients. Qualitative analysis is always subject to unconscious bias on the part

426 of those who perform it, and the psychological background of the two researchers, one a reader

427 in clinical, educational and health psychology and the other a psychology graduate, both of 
428 whom were known to the participants, doubtless informed understanding of the material.

429 However, the quotations themselves demonstrate the high level of psychological literacy, and

430 the extent of self-critical reflection, of the four physiotherapists involved. Additionally, while to

431 a large extent spontaneous, the physiotherapist explanations of both behaviour and intentions

432 might have been somewhat censored for better self-presentation. Thematic analysis was used

433 as given the breadth of the phenomenon being studied a true phenomenological approach was not

434 possible. Lastly, The $\mathrm{N}$ for this study is very small, partly because we wished to be certain of the

435 level of training and experience of our subjects, and partly because the methods themselves

436 were very time-consuming for researchers and for physiotherapist participants. Ideally, a better

437 resourced study would sample a wider range of physiotherapists at work, to achieve data

438 saturation and perhaps use random or purposive sampling of videoed material to make

439 methods feasible for larger numbers.

441 Conclusion

443 This research sought to identify what physiotherapists actually do in practice that qualifies as

444 psychologically informed practice. The analysis here can enrich our understanding of

445 psychological competences in the practice of physiotherapy by providing clinical examples of

446 the application of psychologically informed approaches. Whilst development of generic

447 therapeutic and basic CBT competences form part of physiotherapy undergraduate and early

448 career training,the question is: what additional behavioural changes are required in order to

449 deliver psychologically informed physiotherapy, and promote patient self-management [5]? This 
450 study describes behaviours and techniques used by physiotherapists experienced in

451 psychologically informed aproaches andworking with groups of patients with chronic pain. The

452 physiotherapists studied clearly demonstrated competences as outlined in the model for

453 cognitive and behavioural therapy (CBT) practitioners (table 1) in a clinical setting. They were

454 able to identify and reflect on the skills, applied specifically to the needs and difficulties of

455 people with chronic pain. This paper complements current recommendations for training

456 physiotherapists [13] which focus on development of a treatment manual, workshops, and

457 supervision by experienced CBT practitioners, by providing examples of these skills being used

458 in clinical practice..

Table 4: Themes, sub-themes and illustrative quotes

461

462

Table 5: Additional physio comments: Acknowledging Risks and Boundaries

Ethical Approval: NHS ethics was dealt with by the special office at UCL for NHS related ethics. Approval was obtained; Ref 12/0078. Full ethics was not required as this research did not directly interview patients.

Conflict of Interest: The authors of this paper have no known conflicts of interest.

\section{Reference list:}

1. Fayaz A, Croft P, Langford RM, et al Prevalence of chronic pain in the UK: a systematic review 
and meta-analysis of population studies BMJ Open 2016;6:e010364. doi: 10.1136/bmjopen2015-010364

2. Main CJ, George SZ. Psychosocial Influences on Low Back Pain: Why Should You Care. Phys Ther. 2011;91: 609-613. pmid:21531941

3. Donaldson L. 150 years of the Annual Report of the Chief Medical Officer: On the state of public health 2008. London: Department of Health, 2009.

4. Harding V, Williams A.C.de.C. Extending physiotherapy skills using a cognitive psychological approach. Cognitive behavioural management of chronic pain. Physiotherapy. 1995;81:681688.

5. Matthews J, Hall AM, Hernon M, et al. A brief report on the development of a theoreticallygrounded intervention to promote patient autonomy and self-management of physiotherapy patients: face validity and feasibility of implementation. BMC Health Serv Res 2015;15:260. doi:10.1186/s12913-015-0921-1

6. National Institute for Health and Care Excellence. UK. Low back pain and sciatica in over 16s: assessment and management. November 2016. https://www.nice.org.uk/guidance/ng59

7. Cherkin D, Balderson B, Brewer G, et al. Evaluation of a risk-stratification strategy to improve primary care for low back pain: the MATCH cluster randomized trial protocol. BMC Musculoskeletal Disorders. 2016;17(1):361.

8. van Rensburg J, Rau A, Fourie A, Bracke P, Piet. (2016). Power and Integrated Health Care: Shifting from Governance to Governmentality. International journal of integrated care. 16. 1-11. 10.5334/ijic.2480.

9. Caldwell, K. \& Atwal, A. (2003). The problems of interprofessional healthcare practice in hospitals. British journal of nursing (Mark Allen Publishing). 12. 1212-8.

10.12968/bjon.2003.12.20.11844.

10. McLoughlin, R. Petersen, K. Brook, S. (2015) Physiotherapy in Assessment and Management of Pain, In: Abdominal and Pelvic Pain From Definition to Best Practice, IASP Press. pg 229-238

11. Wilson S, Chaloner N, Osborn M, et al. Psychologically informed physiotherapy for chronic pain: patient experiences of treatment and therapeutic process. Physiotherapy. 2016;103(1):98-105

12. Guerrero A.V.S., Maujean A., Campbell L., Sterling M.. A systematic review and meta-analysis of the effectiveness of psychological interventions delivered by physiotherapists on pain, disability and psychological outcomes in musculoskeletal pain conditions. PROSPERO 2015 CRD42015024444 Available from: http://www.crd.york.ac.uk/PROSPERO/display record.php?ID=CRD42015024444

13. Keefe FJ, Main CJ, George SZ. Advancing psychologically informed practice for patients with persistent musculoskeletal pain: promise, pitfalls, and solutions. Phys Ther . 2018;98:398-407. 
14. Pincus T, Holt N., Vogel S., et al. (2013). Cognitive and affective reassurance and patient outcomes in primary care: a systematic review. Pain 2013154, pp 2407-2416

15. Michie S, Atkins L, West R. The behaviour change wheel. A guide to designing interventions. 1st ed. Great Britain: Silverback Publishing. 2014.

16. Beissner K, Reid MC. Reply. Physical Therapy. 2009;89(5):472-3

17. Sowden G, Hill JC, Konstantinou K, Khanna M, Main CJ, Salmon P, Somerville S, Wathall S, Foster NE. IMPaCT back study team. Targeted treatment in primary care for low back pain: the treatment system and clinical training programmes used in the IMPaCT back study (ISRCTN 55174281). Fam Pract. 2012;29(1):50-62.

18. Hansen Z., Daykin A., Lamb S.E., A cognitive-behavioural programme for the management of low back pain in primary care: a description and justification of the intervention used in the Back Skills Training Trial (BeST; ISRCTN 54717854), In Physiotherapy, Volume 96, Issue 2, 2010, Pages 87-94, ISSN 0031-9406, https://doi.org/10.1016/j.physio.2009.09.008.

19. Physiotherapy Pain Association in collaboration with the Chartered Society of Physiotherapy. Physiotherapy framework - entry level graduate to expert. PPA October 2014. [Accessed 20/11/2018] Available from; https://ppa.csp.org.uk/documents/ppa-physiotherapyframework-entry-level-graduate-expert-describing-values-behaviours.

20. Fuentes J., Armijo-Olivo S., Funabashi M., Miciak M., Dick B., Warren S., Rashiq S., Magee D.J., Gross D.P.; Enhanced Therapeutic Alliance Modulates Pain Intensity and Muscle Pain Sensitivity in Patients With Chronic Low Back Pain: An Experimental Controlled Study, Physical Therapy, Volume 94, Issue 4, 1 April 2014, Pages 477-489,

21. Lewis, M., Morley, S., van der Windt, D. A.W.M., Hay, E., Jellema, P., Dziedzic, K. and Main, C. J. (2010), Measuring practitioner/therapist effects in randomised trials of low back pain and neck pain interventions in primary care settings. European Journal of Pain, 14: 1033-1039. doi:10.1016/j.ejpain.2010.04.002

22. Synnott $A, O^{\prime}$ Keeffe $M$, Bunzli S, et al. Physiotherapists may stigmatise or feel unprepared to treat people with low back pain and psychosocial factors that influence recovery: a systematic review. J Physiother. 2015;61(2):68-76. Medline:25812929 http://dx.doi.org/10.1016/j.jphys.2015.02.016.

23. Yuguero O, Marsal JR, Buti M, Esquerda M, Soler-González J. Descriptive study of association between quality of care and empathy and burnout in primary care. BMC Medical Ethics. 2017;18:54. doi:10.1186/s12910-017-0214-9.

24. Kerasidou A, Horn R. Making space for empathy: supporting doctors in the emotional labour of clinical care. BMC Medical Ethics. 2016;17:8. doi:10.1186/s12910-016-0091-7. 
25. Martin EB, Mazzola NM, Brandano J, Luff D, Zurakowski D, Meyer EC. Clinicians' recognition and management of emotions during difficult healthcare conversations. Patient Educ Couns. 2015;98(10):1248-54. doi: 10.1016/j.pec.2015.07.031.

26. Driver C, Kean B, Oprescu F, Lovell GP. Knowledge, behaviors, attitudes and beliefs of physiotherapists towards the use of psychological interventions in physiotherapy practice: a systematic review. Disabil Rehabil. 2017 Nov;39(22):2237-2249. doi: 10.1080/09638288.2016.1223176.

27. Chester E., Robinson NC., Roberts LC., Opening clinical encounters in an adult musculoskeletal setting, In Manual Therapy, Volume 19, Issue 4, 2014, Pages 306-310, ISSN 1356-689X, https://doi.org/10.1016/j.math.2014.03.011.

28. Roberts LC., Whittle CT., Cleland J., Wald M.; Measuring Verbal Communication in Initial Physical Therapy Encounters, Physical Therapy, Volume 93, Issue 4, 1 April 2013, Pages 479-491, https://doi.org/10.2522/ptj.20120089

29. Main CJ, Sowden G, Hill JC, Watson PJ, Hay EM. 2012. Integrating physical and psychological approaches to treatment in low back pain: the development and content of the STarT Back trial's 'high-risk' intervention (StarT Back; ISRCTN 37113406). Physiotherapy, vol. 98(2), 110-116.

30. Singh, A., Klapper, A., Jia, J., Fidalgo, A., Tajadura-Jiménez, A., Kanakam, N., Bianchi-Berthouze, N., Williams, A. (2014). Motivating People with Chronic Pain to Do Physical Activity: Opportunities for Technology Design. Proceedings of the SIGCHI Conference on Human Factors in Computing Systems, 2803-2812.

31. Singh, A., Berthouze-Bianchi, N. L., \& Williams, A. (2017). Supporting Everyday Function in Chronic Pain Using Wearable Technology. CHI '17 Proceedings of the $2017 \mathrm{CHI}$ Conference on Human Factors in Computing Systems. ACM. doi:10.1145/3025453.3025947

32. Singh, A., Piana, S., Pollarolo, D., Volpe, G., Varni, G., Tajadura-Jimenez, A., Bianchi-Berthouze, N. (2016). Go-with-the-flow: Tracking, Analysis and Sonification of Movement and Breathing to Build Confidence in Activity Despite Chronic Pain. Human-Computer Interaction, 31 (3-4), 335383.

33. Talvitie, U. (2000). Socio-affective chatracteristics and properties of extrinsic feedback in physiotherapy. Physiotherapy Research International, 5(3); 173-88.

34. Ickes W. Hall JA, Bernieri FJ. Measuring empathic accuracy, Interpersonal sensitivity: Theory and measurement. , 2001 Mahwah, NJ Erlbaum (pg. 219 -241)

35. Braun V, Clarke V. Using thematic analysis in psychology. Qual Res Psychol. 2006; 3(2):77-101.

36. Ritchie J, Spencer L, O'Connor W. Carrying out Qualitative Analysis. In: Ritchie J, Lewis J, editors. Qualitative research practice: A guide for social science students and researchers. London: Sage Publications Ltd; 2003: 219-262. 
37. Foster NE, Anema JR, Cherkin D, et al. Prevention and treatment of low back pain: evidence, challenges, and promising directions. Lancet 2018; published online March 21.

http://dx.doi.org/10.1016/S0140-

6736(18)30489-6.

38. Bennett-Levy, J., Butler, G., Fennell, M., Hackman, A., Mueller, M., \& Westbrook, D. (Eds.). (2004).

Oxford guide to behavioural experiments in cognitive therapy. Cognitive behaviour therapy:

Science and practice series. http://dx.doi.org/10.1093/med:psych/9780198529163.001.0001 